\title{
OBSERVATION OF ERUPTIVE EVENTS WITH THE SIBERIAN RADIOHELIOGRAPH
}

\author{
A.Yu. Fedotova \\ Institute of Solar-Terrestrial Physics SB RAS, \\ Irkutsk, Russia, fedotovanastya@iszf.irk.ru
}

\section{A.T. Altyntsev}

Institute of Solar-Terrestrial Physics SB RAS, Irkutsk,Russia,altyntsev@iszf.irk.ru

\section{A.A. Kochanov}

Institute of Solar-Terrestrial Physics SB RAS, Irkutsk, Russia, kochanov@iszf.irk.ru

\author{
S.V. Lesovoi \\ Institute of Solar-Terrestrial Physics SB RAS, \\ Irkutsk,Russia,lesovoi@iszf.irk.ru
}

\author{
N.S. Meshalkina \\ Institute of Solar-Terrestrial Physics SB RAS, \\ Irkutsk,Russia,nata@iszf.irk.ru
}

\begin{abstract}
We describe methods for monitoring eruption activity with the first phase of the multiwave Siberian Radioheliograph (SRH-48). We give examples of the recorded eruptive events: 1) rise of a prominence above the limb observed in the radio map sequence of April 24, 2017; 2) a jet recorded on August 2, 2017, whose cold matter screened a compact microwave source for several tens of minutes. The shading due to the jet appearance was observed on SRH-48 correlation curves as the so-called "negative" burst. Using the "negative" burst on the correlation curves of February 9, 2017 as an example, we show that the intervals with depression of
\end{abstract}

the microwave emission of local sources are not always caused by shading of their emission. In this event, the radio brightness decreased within ten hour period of the increased quasi-stationary emission during the development of AR 12635 magnetic structure. Similar behavior was observed in EUV, SXR, and radio emission at $17 \mathrm{GHz}$.

Keywords: radioheliograph, Sun, eruptive events, jet.

orbit. If CME is earthward, it reaches Earth within one or three days disturbing near-Earth plasma, thereby being an important factor of space weather.

The occurrence of CMEs is often associated with filament eruption. Analyzing the sequence of NoRH images at $17 \mathrm{GHz}$ and using extensive statistical material. Gopalswamy et al. [2003] have established that $82 \%$ of filament eruptions were accompanied by CMEs. Filaments contain cold plasma with a brightness temperature of about $8000 \mathrm{~K}$, hence CME is optically thick in microwaves. In the emission of a filament, thermal bremsstrahlung predominates, it therefore shows as a dark structure against the disk whose brightness temperature is $10000 \mathrm{~K}$ at $17 \mathrm{GHz}$. Above the limb, the filament stands out against the sky [Kundu et al., 2004]. In SSRT images, filament and quiet solar disk brightness temperatures are more contrast than those in NoRH images. This is due to the fact that the brightness temperature of the quiet Sun at $5.7 \mathrm{GHz}$ is higher $(16000 \mathrm{~K})$. The first observations of erupting filaments with SSRT have been reported by Uralov et al. [2002] and Alissandrakis et al., [2013].

Along with large-scale filament eruptions, EUV observations often reveal frequently occurring small-scale plasma ejections into the solar corona, the so-called jets. They are rapidly rising collimated plasma streams along magnetic field lines, observed in EUV and HXR [Shimojo et al., 1996]. Their length can vary from small jet size characteristic of macrospicules to hundreds of thousands of kilometers for X-ray jets [Raouafi et al., 2016], and their lifetime can vary from several minutes to several hours. Observations of jets in microwave emission are extremely 
rare due to their small size and relatively low brightness temperatures. There are well-known papers [Kundu et al., 1999; Nakajima, Yokoyama, 2002] that describe observations of events related to the jet at $17 \mathrm{GHz}$. It has been shown that jet plasma can be heated up to a brightness temperature of $28 \mathrm{MK}$ and can also feature a non-thermal electron component. Studies of the nature of jets are important for understanding coronal heating and solar wind formation processes, i.e. they deal with a wide range of heliophysical problems.

In the microwave emission, mass ejections including small jets can also be detected from the shading of a local microwave source by a cloud of cold absorbing substance. In light curves of microwave emission, the shadings show up as depressions, the so-called negative bursts, lasting from several minutes to tens of minutes. The first event with a negative burst is described by Covington [1969] as a post-burst decrease in the radio flux at $2.8 \mathrm{GHz}$ after the impulsive radio burst on May 19,1951 . In recent years, some researchers have studied the diagnostic potential of negative bursts, using radioheliograph observations [Maksimov and Nefedjev, 1991; Grechnev et al., 2013, 2014; Uralov et al., 2014].

In 2016, the ISTP SB RAS Radioastrophysical Observatory began observations with the Multiwave Siberian Radioheliograph (SRH) in a frequency range 4-8 GHz [Lesovoi, Kobets, 2017]. The first observations showed that correlation curves make it possible to reliably detect events with behind-the-limb eruptions and negative bursts [Lesovoi, Kobets, 2017; Grechnev et al., 2018]. The main purpose of this paper is to continue to explore SRH capabilities to observe eruptive phenomena. We consider two methods of observing plasma ejections. The first uses constructed images to trace the movement of eruptive filaments and jets over the solar limb. The second method employs solar disk images captured with SRH to study the dynamics of the source the shading of which causes a negative burst of microwave emission.

\section{OBSERVATIONS}

\section{Instruments}

The Siberian Radioheliograph was developed by application of SSRT rotators and infrastructure [Lesovoi, Kobets, 2017b]. The purpose of the modernization: 1) to implement the aperture synthesis of images, which enables us to substantially improve temporal resolution, 2) to turn to multiwave measurements in the frequency range 4-8 GHz. The main characteristics of the first stage of the radioheliograph with 48 antennas and $152 \mathrm{~m}$ maximum base: the receiving frequency varies, according to the protocol, cyclically in the operating frequency range 4-8 GHz; the spatial resolution depending on frequency is within $0.8^{\prime}-1.6^{\prime}$; the spectral resolution of the frequency channel is $10 \mathrm{MHz}$; the flux sensitivity is $\sim 100 \mathrm{Jy}$. Both circular polarized components (RCP and LCP) are measured.

We have made the observations at 4.2, 4.5, 5.2, 6.0, and $6.8 \mathrm{GHz}$ and selected events for the analysis, using correlation curves available on the website [http://badary.iszf.irk.ru]. The correlation curves are plotted by summing complex correlations calculated for different pairs of antennas, and allow us to observe variations in received radio emission. Their calculations are described in detail in [Lesovoi et al., 2017].

To analyze the events, we have used sequences of microwave images acquired with the Nobeyama Radioheliograph at $17 \mathrm{GHz}$ [http://solar.nro.nao.ac.jp/], EUV images from the Solar TErrestrial RElations Observatory (STEREO) [https://stereo.gsfc.nasa.gov; Kaiser et al., 2007] and Interface Region Imaging Spectrograph (IRIS) [http://www.lmsal.com/solarsoft/irisa/; Culhane et al., 2007; Pontieu et al., 2014].

\section{April 24, 2017 eruptive filament}

SRH began observations in solar minimum, during which events with above-the-limb manifestations of eruptions were rare. One of the few significant events was the April 24, $2017 \mathrm{CME}$ whose frontal structure appeared in LASCO/C2 images at 02:48 UT. SRH managed to detect above the limb (Figure 1, $a-c$ ) the rise of a filament whose eruption was clearly observed by the orbital observatory SDO/AIA at wavelengths of 171 , 304, and $131 \AA$ (Figure $1, b-e$ ).

Figure 1 presents a sequence of images of eruptive filament evolution from 02:00 to 04:00 UT acquired with SRH at frequencies of $6.0(a), 5.2(b)$, and $4.5 \mathrm{GHz}(c)$; with SDO at a wavelength of $304 \AA(d)$, and with NoRH at 17 $\mathrm{GHz}(e)$. At the beginning of the observations, the filament top with coordinates of E40N40 was on the solar disk [https://kauai.ccmc.gsfc.nasa.gov/DONKI/search/].

There is good agreement between positions and heights of the emerging filament in the images in different spectral ranges. The filament top appeared above the limb at about 02:00 UT; by 04:00 UT, it rose above the limb higher than $150 \mathrm{Mm}$. In the SRH images, the filament structure is blurred owing to insufficient spatial resolution, which is proportional to the frequency of observation. It is seen that at $6.0 \mathrm{GHz}$ the images are more contrast. Panels $a$ and $b(5.2$ and $6.0 \mathrm{GHz})$ as well as panel $d(304 \AA)$ show the part of the filament against the solar disk that is absent in the images acquired at 17 GHz. This is due to the fact that the optical thickness of the filament in the SRH range is by an order of magnitude higher than that at $17 \mathrm{GHz}$. The darkening of the filament against the solar disk indicates that its brightness temperature is lower than the effective temperature of the quiet Sun $(16000 \mathrm{~K})$ at $6.0 \mathrm{GHz}$ [Zirin et al.,1991; Borovik, 1994]. At lower frequencies, the filament is less pronounced against the background of the solar disk, since the relative difference between effective temperatures of the filament and solar disk decreases. In general, the sensitivity of SRH receiving systems allows us to detect an increase in the total solar emission associated with the appearance of behind-the-limb sources. After the second stage of SRH with antennas located in outer SSRT posts is activated, the spatial resolution will increase several times and become sufficient to study structural features of eruptive ejections, plasma density and brightness temperature distribution in erupting filaments. 


\subsection{GHz SRH Stokes R}
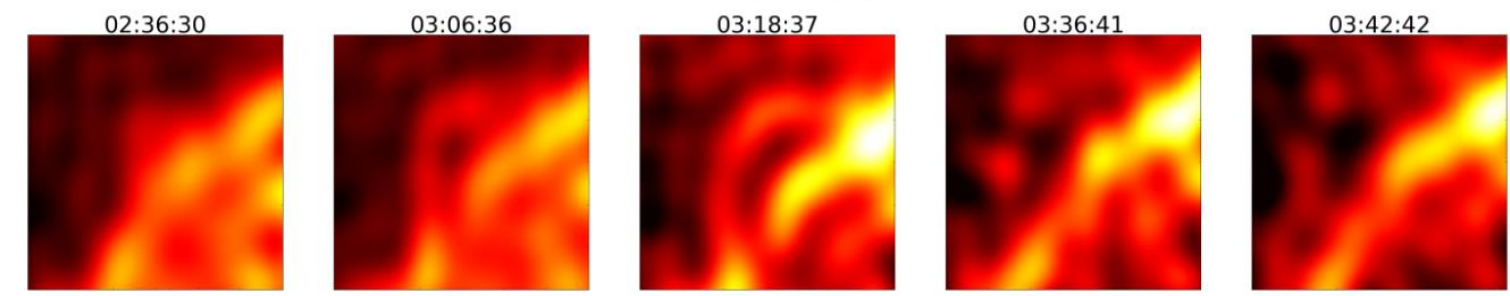

5.2 GHz SRH Stokes R
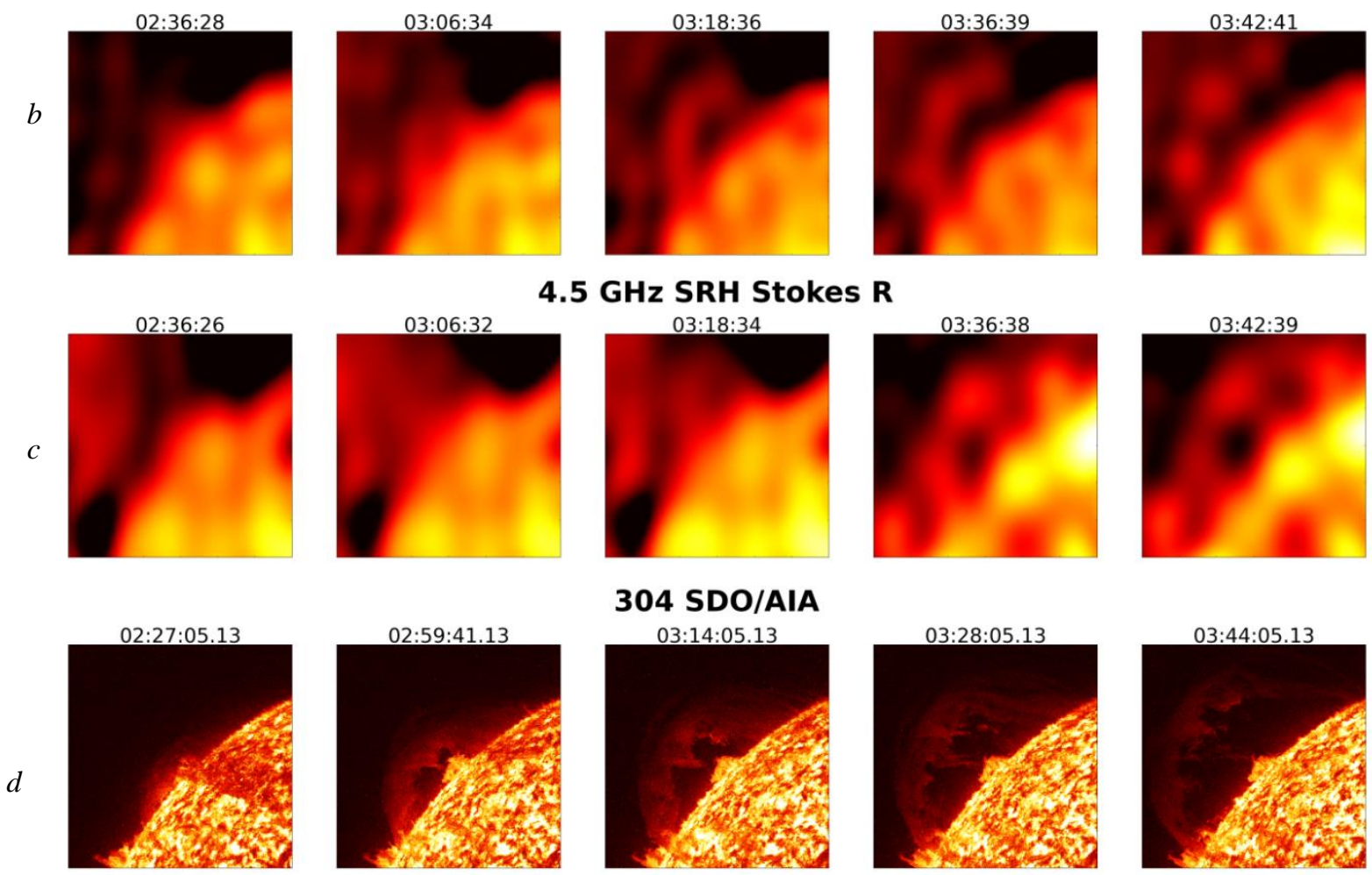

\section{SDO/AIA}
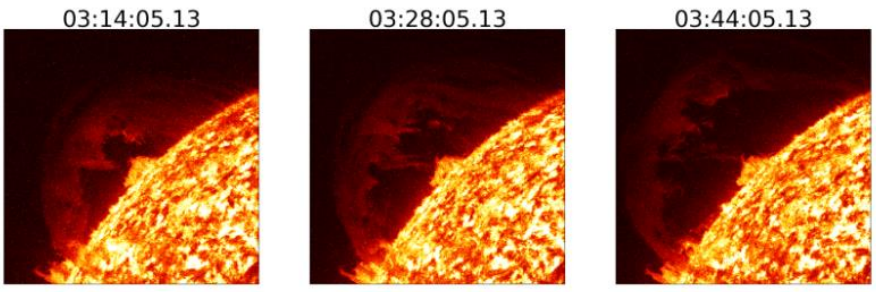

\section{GHz NoRH}
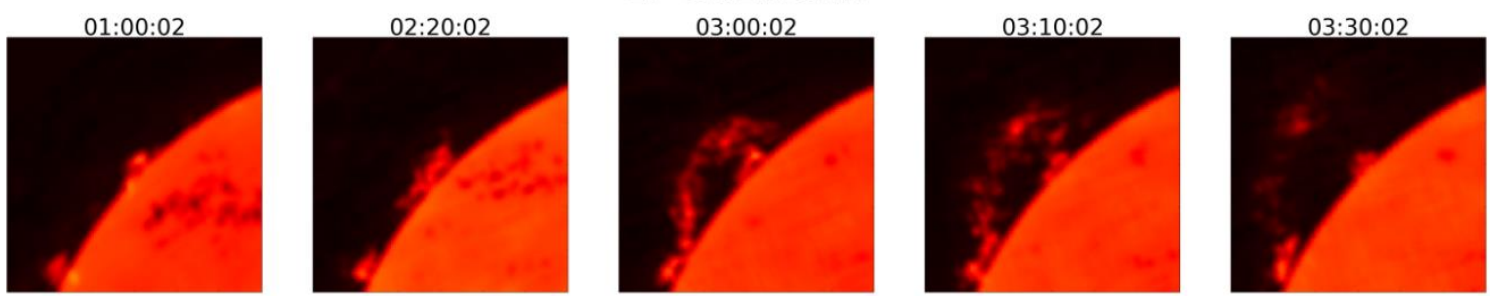

Figure 1. Sequence of images from SRH at $6.0,5.2$, and $4.5 \mathrm{GHz}(a-c)$, from SDO at a wavelength of $304 \AA(d)$, and from $\mathrm{NoRH}$ at $17 \mathrm{GHz}(e)$ during the appearance of the eruptive filament above the limb on April 24, 2017. The frame size is $600^{\prime \prime} \times 600^{\prime \prime}$

In the correlation curves, the response to the emergence of the eruptive filament is weak. The top panel (Figure 2) shows the difference between the correlation curves on April 24 and 25, 2017, measured on adjacent days when the Sun was active and quiet respectively. It allows us to determine the contribution of active processes and reduce the influence of the diurnal variation of the curves, caused by the change of the interferometer bases with Earth revolution about the Sun. The bottom panel depicts the microwave emission flux at $17 \mathrm{GHz}$ from the above-the-limb filament, derived from sequences of NoRH images by subtracting the solar disk flux.

The SRH correlation curves show the microwave emission enhancement between 1:00 and 02:00 UT, which is a response to the B2.4 flare in the active region 2653 (S11E51), located away from the filament eruption. The appearance of the filament above the limb can be attributed to the slowing of the decrease on the curves for high frequencies from 02:00 to 04:00 UT.

\section{August 2, 2017 jet}

Let us examine the second method for detecting eruptive ejections, using the August 2, 2017 event as an example. The SRH correlation curves have several daytime intervals (Figure 3) with brightness depression (negative bursts). The events are weak, and there are no responses on the SDO/AIA EUV light curves. The IRIS data 

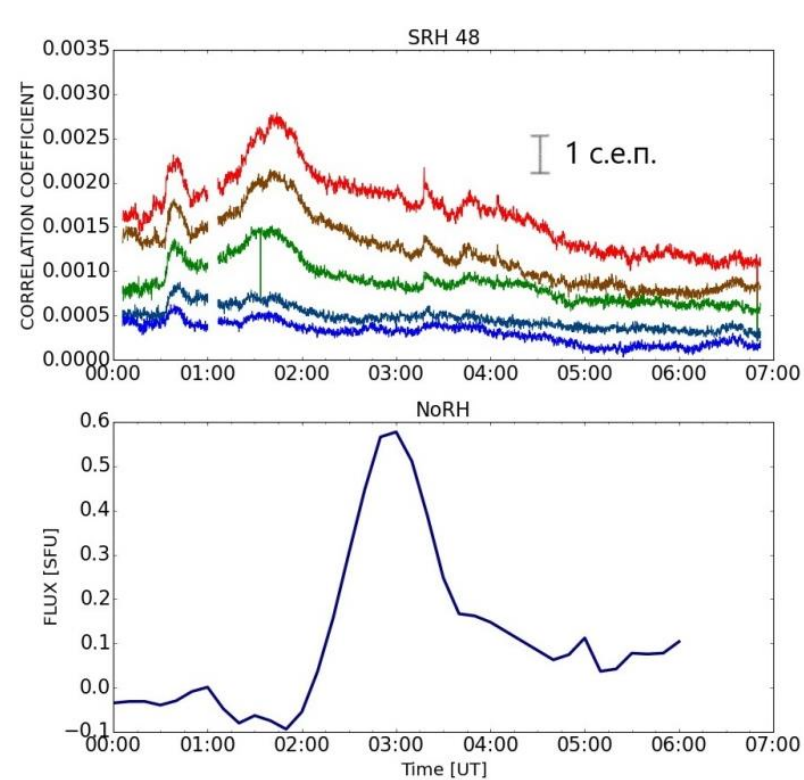

Figure 2. Top: difference between April 24 and 25 correlation curves, measured with $\mathrm{SRH}$ in the $\mathrm{R}+\mathrm{L}$ intensity from 00:00 to 07:00 UT. The length of the vertical line corresponds to $1 \mathrm{sfu}$. The curves correspond to frequencies of 4.2, 4.5, 5.2, 6.0 , and $6.8 \mathrm{GHz}$ (from top to bottom). Bottom: variation in the above-the-limb flux of microwave emission at $17 \mathrm{GHz}$

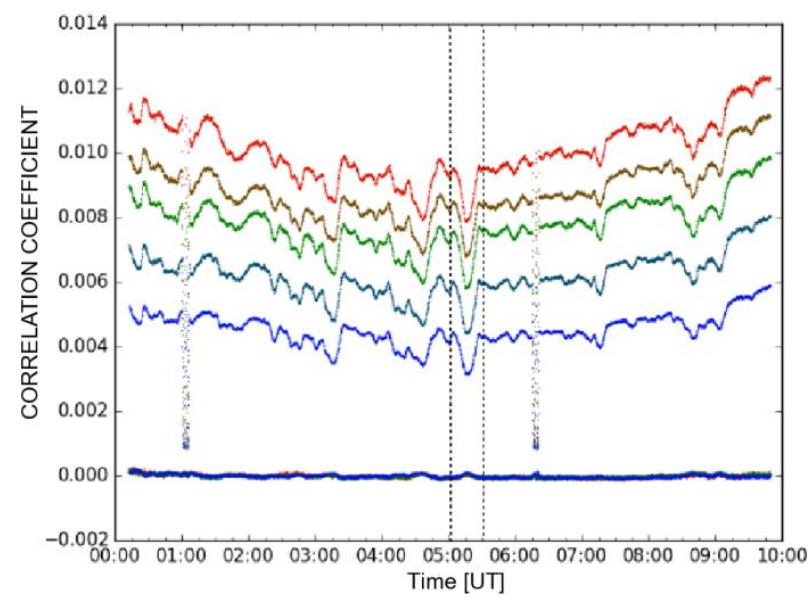

Figure 3. August 2, 2017 correlation curves. The upper correlation curves are the intensity at 4.2, 4.5, 5.2, 6.0, and 6.8 $\mathrm{GHz}$ (from top to bottom); the lower ones with near-zero values show circular polarization. The interval with dispersion from 05:00 to 05:30 UT, during which IRIS SJI observed a jet (vertical dashed lines)

on ultraviolet emission were available for the upgoing active region 12670 from 04:49 to 06:18 UT. It has been found that the depression of the microwave emission from 05:00 to 05:30 UT was observed during the jet recorded in the FUV 1332-1406 $\AA$ range (Figure 4). The jet is designated by a blue circle. In IRIS SJI image sequences there is a rapidly increasing bright jet rising above the active region. In Figure 3, the corresponding time interval with the depression is indicated by vertical lines. The depth of the depression at all SRH frequencies is roughly the same and corresponds to a decrease in solar radio brightness by a few sfu.

Figure 5 presents a magnetogram of the region in which the jet occurred. On the magnetogram, contours

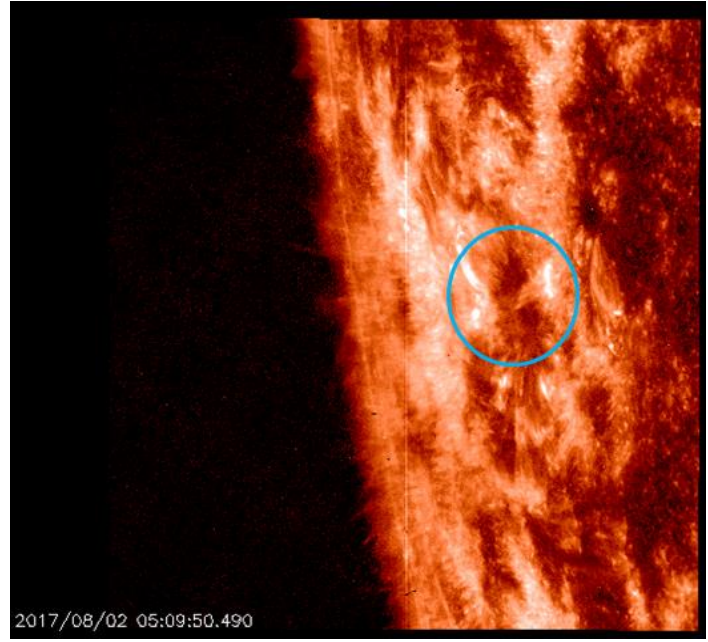

Figure 4. Jet of August 2, 2017, 05:09 UT as derived from IRIS SJI data in the FUV band (1330 $\AA)$. The frame size is $400^{\prime \prime} \times 400^{\prime \prime}$

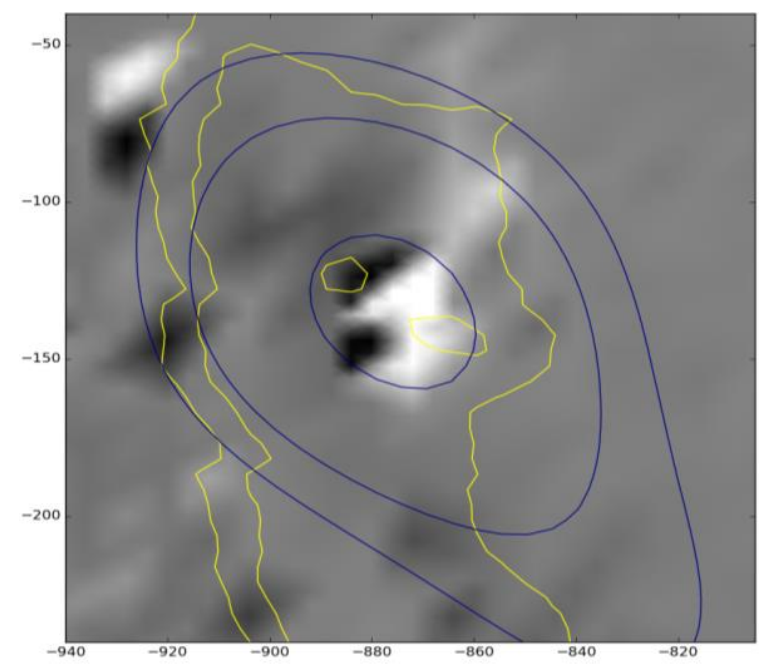

Figure 5. Magnetogram with contours of a source according to data from SRH at $6.0 \mathrm{GHz}$ (blue contours), from NoRH at $17 \mathrm{GHz}$ (yellow contours) at 05:20 UT. Contour levels are $0.3,0.5$, and 0.9 of maxima on intensity maps

show microwave emission brightness distribution at 6.0 and $17 \mathrm{GHz}$. The resolution of the Nobeyama Radioheliograph is $10^{\prime \prime}$ and allows us to resolve two brightness centers corresponding to $\mathrm{N}$ and $\mathrm{S}$ magnetic field polarities.

In general, the emission at the 0.5 beamwidth covers the entire active region. The SRH resolution is much lower than $80^{\prime \prime}$, and the source structure is not resolved. The source at $6.0 \mathrm{GHz}$, as well as at $17 \mathrm{GHz}$, is extended from north to south and its apparent FWHM size is $80^{\prime \prime} \times 150^{\prime \prime}$. SRH maps in circular polarization show that the microwave source is left-handed polarized. Thus, the interval with partial shading of the source appears on correlation curves as an increase in the right-handed polarized emission. According to the IRIS SJI images, the jet plasma propagated eastward in the plane of the sky, i.e. it shaded the region with the photospheric magnetic field directed from the observer. In this case, the shading should cause the ordinary-mode emission to decrease, which is consistent with the appearance of the ejection with the sign of ordinary wave on the correlation curves. 


\section{Brightness depression on February 09, 2017}

CME can be detected as a negative burst on correlation curves in case of shading of a local bright source or for a sufficiently large area of shading of the quiet Sun. However, in some cases, negative bursts are a response to a sharp decrease in the quasi-stationary emission of the developing active region rather than to the shading. Sometimes, long-term active processes in active regions may occur as an increase in the quasi-stationary level of microwave emission for several hours, with a subsequent sharp decrease. A perfect example is events of February 08 and 09, 2017 (Figure 6). The difference is shown between the correlation curves obtained by subtracting values acquires on the quiet day of February 8 with no significant correlation variations from the curves of February 9. There is a pronounced synchronous decrease in the radio flux at all frequencies between 04:30-05:45 UT, which is indicated by vertical lines on the intensity correlation curves. The relative depth of the depression on the correlation curves is approximately the same at all frequencies (up to $1.5 \%$ ), which corresponds to a decrease in the solar flux by several sfu. The polarization correlation curves do not show a response to the negative burst in intensity. It should be noted that on the light curves of total flux of the $2-24 \mathrm{GHz}$ spectropolarimeter there is no response to this negative burst. This confirms that the depression has a small depth.

The radio maps of the full solar disk obtained with SRH-48 allowed us to determine the solar region a brightness decrease in which leads to the depression on the correlation curves. Figure $7, a, b$ presents sequences of radio maps at $6.8 \mathrm{GHz}$ for RCP and LCP in the upper and lower rows respectively. On the solar disk there is one bright unpolarized source with coordinates $\left(320^{\prime \prime},-400^{\prime \prime}\right)$. The source emission intensity is much lower in the second column of the maps, which corresponds to the time of the correlation curve dip. The microwave source represents a rapidly developing active region NOAA 12635.

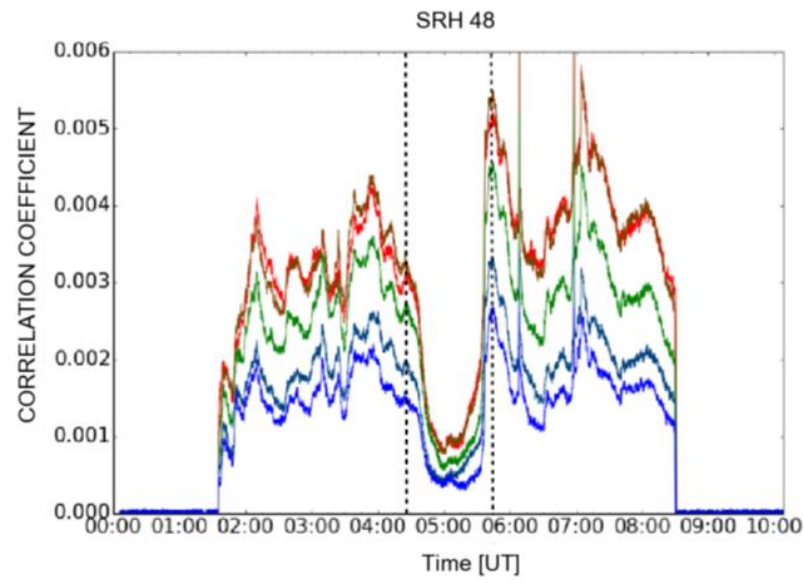

Figure 6. Difference between the correlation curves for February 8, 9, 2017 with depression from 04:30 to 05:45 UT. The curves correspond to 4.2, 4.5, 5.2, 6.0, and $6.8 \mathrm{GHz}$ (from top to bottom)
To figure out the causes of the microwave source brightness decrease, we examine the emission dynamics in other ranges. Figure 8 presents light curves of solar emission from 00:00 to 13:00 UT on February 9 during the formation of the active region NOAA 12634. The top panel depicts the dependence of the brightness temperature at the source maximum at 6.0 GHz. The radioheliograph has been calibrated on the assumption that the brightness temperature of the solar disk at $6.0 \mathrm{GHz}$ is $16000 \mathrm{~K}$ [Borovik, 1994].

It can be seen that during the depression the brightness temperature falls from 300000 to $90000 \mathrm{~K}$. Note that because of insufficient spatial resolution, these estimates may be underestimated.

Figure 8, $b$ depicts a similar dependence at $17 \mathrm{GHz}$. Triangles indicate values derived from full disk radio maps in cadence of one map per $10 \mathrm{~min}$; the solid line, in cadence of map/s. If such a significant level of depression at $17 \mathrm{GHz}$ was due to the shading material above the source, at $6 \mathrm{GHz}$ the source would be completely shaded rather than being seen as in Figure 7, $a, b$. The radio maps at $17 \mathrm{GHz}$ also show a source with apparent size of the order of the NoRH beam, i.e. 10".

Continuous observations of the developing region can be made with GOES and SDO. The emission intensity

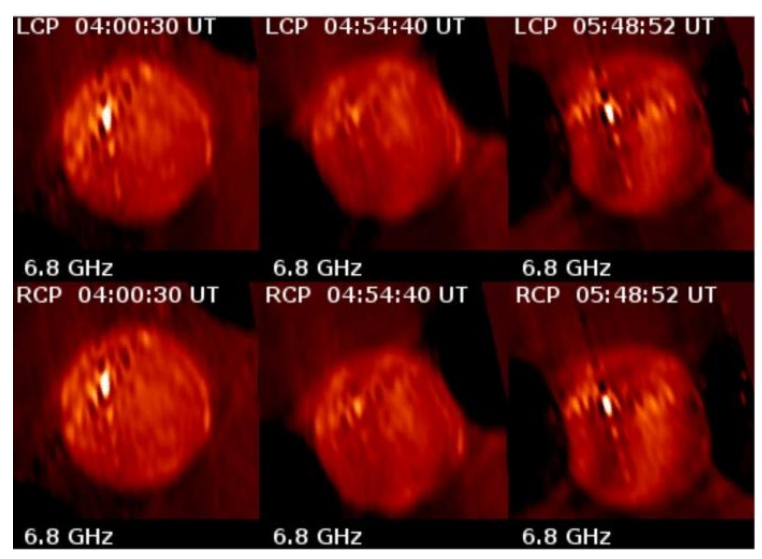

Figure 7. Sequences of SRH radio maps at $6.8 \mathrm{GHz}$ during depression on the correlation curves in left (LCP) and right (RCP) circular polarization ( $a, b$ respectively)

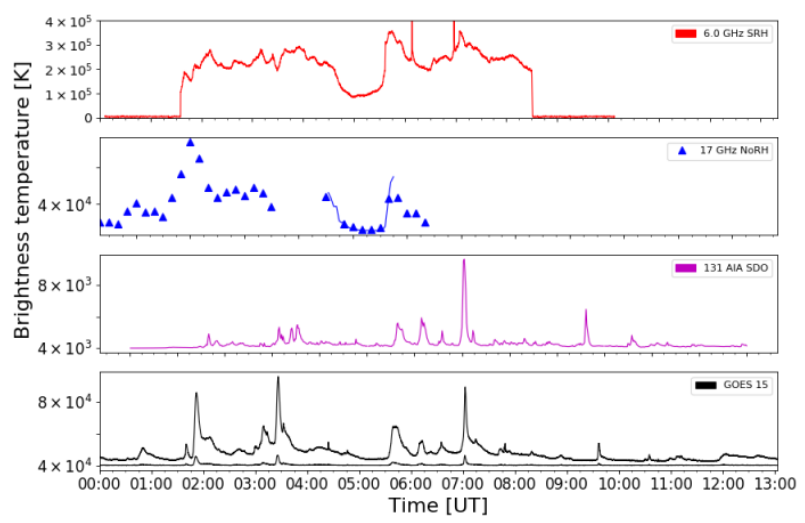

Figure 8. Solar activity variations on February 9 from 00:00 to 07:00 UT, according to data from SRH the maximum brightness temperature is at $6.0 \mathrm{GHz}$; from NoRH, at $17 \mathrm{GHz}$; SDO signal value at a wavelength of $131 \AA$; GOES 15 fluxes in two SXR channels 
in EUV in the highly ionized iron line of $131 \AA$ after 20:00 UT gradually increases until 07:00 UT, and then decreases until 13:30 UT on February 9. The SXR behavior is similar. In both the emission ranges, the period of microwave emission depression corresponds to low emission levels in EUV and SXR.

In SDO/AIA EUV images, we did not find plasma streams shading NOAA 12634 . Thus, we can confidently assert that this negative burst is not connected with the cold plasma eruption - it is a response to the dynamics of active processes of energy release in the active region.

\section{DISCUSSION}

Observations of eruptive events with SRH having 48 antennas and $154 \mathrm{~m}$ maximum base demonstrate that the radioheliograph has sufficiently high sensitivity for detecting eruptive events including weak jets on the solar disk.

The traditional method is to observe microwave sources appearing above the limb. An example of the use of the observations with a microwave coronagraph is the cata$\log$ of limb events at $17 \quad \mathrm{GHz}$ [http://solar.nro.nao.ac.jp/norh/html/prominence/], which has been available on the Nobeyama Radioheliograph website since 1992. The analysis of observations of hundreds of prominences at $17 \mathrm{GHz}$ during solar cycle 23 has shown that the frequency of their occurrence is similar to the variation in the number of sunspots, although times of peaks differ. The frequency of occurrence of prominences depends on latitude, and the region of their occurrence migrates with solar cycle phase [Shimojo et al., 1996]. The relationship of flare activity to plasma eruption is shown in the catalog [https://hinode.isee.nagoya-u.ac.jp/ICCON/]. A similar monitoring at $4-8 \mathrm{GHz}$ is being performed using $\mathrm{SRH}$ data. The complexity of the current observations with SRH is the insufficient spatial resolution, which will be increased by several times within one to two years with commissioning of remote antennas. The experience of SSRT observations at $5.7 \mathrm{GHz}$ has shown that the resolution of about $10^{\prime \prime}-15^{\prime \prime}$ will allow us to determine kinematic characteristics of CME structures up to several solar radii, thereby filling the gap between SDO/AIA EUV and LASCO/C2 scattered light observations [Uralov et al., 2002; Alissandrakis et al., 2013].

The comparison between images of erupting filaments at different frequencies will permit us to use an independent method to check brightness temperature measurements made from EUV data and extend the range of its measurements to lower values.

The study of jets and their related phenomena is important for space weather forecast because jets are among the most frequent manifestations of solar activity and are connected with processes of corona heating and solar wind formation. From the observations it follows that the monitoring of negative bursts is valuable for diagnostics of parameters of jets appearing on the solar disk. The analysis techniques have been developed based on deep microwave emission depressions revealed by observations of the integral solar flux. Kuzmenko et al. [2009] have demonstrated that values of radio absorption, measured in the integral microwave emission at several frequencies, can be employed to estimate absorbing plasma parameters such as brightness temperature, optical thickness, area of a shading screen and its height above the chromosphere. However, an unambiguous determination of causes of negative bursts, as is shown by the analysis of the February 9, 2017 event, requires the use of spatially-resolved observations in other wavelengths.

\section{CONCLUSION}

The test observations made with the first stage of SRH have shown that the new instrument designed to study eruptive events can be effectively used both to solve fundamental problems and to monitor solar activity for applied purposes. The potential of multiwave microwave observations will be realized with a several-fold increase in the spatial resolution of SRH, adaptation of the methods for eliminating side lobes of the interferometer from original images to SRH data. The experience with the multiwave radioheliograph, as an upgrade of SSRT, for the 4-8 GHz frequency range has been used in designing a new instrument for the $3-24 \mathrm{GHz}$ frequency range, the construction of which is scheduled for 2018.

We thank sincerely V.F. Melnikov for his assistance in working with NoRH data. Meshalkina N.S. gratefully acknowledges Collaborating Research Programme KLSA201702.

The work was supported by the Russian Science Foundation (project No. 18-12-00172). The experimental data were obtained using the Unique Research Facility Siberian Solar Radio Telescope [http://ckp-rf.ru/usu/73606].

\section{REFERENCES}

Alissandrakis C.E., Kochanov A.A., Patsourkos S., Altyntsev A.T., Lesovoi S.V., Lesovaya N.N. Micro-wave and EUV Observations of an Erupting Filament and Associated Flare and Coronal Mass Ejections. PASJ, 2013, vol. 65. article id. S8 10 pp. DOI: 10.1093/pasj/65.sp1.S8

Borovik V.N. Quiet Sun from multifrequency radio observations on RATAN-600. Solar Phys. 1994. vol. 432, pp. 185-190. DOI: 10.1007/3-540-58041-7_217.

Brueckner G.E., Howard R.A., Koomen M.J., Korendyke C.M., Michels D.J., Moses J.D., Socker D.G., Dere K.P., Lamy P.L., Llebaria A., Bout M.V., Schwenn R., Simnett G.M., Bedford D.K., Eyles C.J. The Large Angle Spectroscopic Coronagraph (LASCO). Solar Phys. 1995, vol. 162, iss. 1-2, pp. 357-402. DOI: 10.1007/BF00733434.

Canfield R.C., Reardon K.P., Leka K.D., Shibata, K., Yokoyama, T., Shimojo, M. H-alpha Surges and X-ray jets in AR 7260. Astrophys. J. 1996, vol. 464, pp. 1016. DOI: 10.1086/177389.

Covington A.E. Solar radio emission at $10.7 \mathrm{~cm}$. Royal Astron. Soc. of Canada. 1969, vol. 63, 125 p.

Culhane J.L., Harra L.K., James A.M., et.al. The EUV Imaging Spectrometer for Hinode. Solar Phys. 2007, vol. 243, iss. 1, pp. 19-61. DOI: 10.1007/s01007-007-0293-1.

Gopalswamy N., Lara A., Yashiro S., Howard R.A. Coronal mass ejections and solar polarity reversal. Astrophys. J. Let. 2003, vol. 598, no. 1, pp. L63-L66. DOI: 10.1086/380430.

Grechnev V.V. A method to analyze imaging radio data on solar flares. Solar Phys. 2003, vol. 213, iss. 1, pp. 103-110. DOI: $10.1023 / \mathrm{A}: 1023213403562$.

Grechnev V.V., Meshalkina N.S., Chertok I.M., et al. Reltions between Microwave Bursts and near-Earth High-Energy Proton 
Enhancements and their Origin. Astronomical Society of Japan. 2013, vol. 65, iss. sp. 1. DOI: 10/1007/s11207-015-0797-6.

Grechnev V.V., Uralov A.M., Chertok I.M., Slemzin V.A., Filippov B.P., Egorov Ya.I., Fainshtein V.G., Afanasyev A.N., Prestage N.P., Temmer M. A challenging solar eruptive event of 18 November 2003 and the causes of the 20 November geomagnetic superstorm. II. CMEs, shock waves, and drifting radio bursts. Solar Phys. 2014, vol. 289, iss. 4, pp. 1279-1312. DOI: $10.1007 / \mathrm{s} 11207-013-0397-2$.

Grechnev V.V., Kiselev V.I., Meshalkina N.S., Chertok I.M. Relations between microwave bursts and near-Earth high-energy proton enhancements and their origin. Solar Phys. 2015, vol. 290, iss. 10, pp. 2827-2855. DOI: 10.1007/s11207-015-0797-6.

Grechnev V.V., Lesovoi S.V., Kochanov A.A., A. M. Uralov, A. T. Altyntsev, A. V. Gubin, D. A. Zhdanov, E. F. Ivanov, G. Ya. Smolkov, L. K. Kashapova Multi-instrument view on solar eruptive events observed with the Siberian Radioheliograph: From detection of small jets up to development of a shock wave and CME. J. Atmosph. Solar-Terr. Phys. 2018, vol. 174, pp. 46-65. DOI: 10.1016/j.jastp.2018.04.014.

Kaiser M.L., Kucera T.A., Davila J.M., Cyr O.C.St., Guhathakurta M., Christian E. The STEREO Mission: An Introduction. Space Sci. Rev. 2007, vol. 136, iss. 1-4, pp. 516.

Kundu M.R., Nindos A., Raulin J.-P., Shibasaki K., White S.M., Nitta N., Shibata K., Shimojo M. A microwave study of coronal ejecta. Astrophys. J. 1999, vol. 520, iss. 1, pp. 391-298. DOI: $10.1086 / 307454$.

Kundu M.R., White S.M., Garaimov V.I., Manoharan P.K., Subramanian P., Ananthakrishnan S., Janardhan P. Radio observations of rapid acceleration in a slow filament eruption / Fast coronal mass ejection event. Astrophys. J. 2004, vol. 607, no. 1, pp. 530-539. DOI: 10.1007/s11214-007-9277-0.

Kuzmenko I.V, Grechnev V.V., Uralov A.M. A study of eruptive solar events with negative radio bursts. Astron. Rep. 2009, vol. 53, iss. 11, pp. 1039-1049. DOI: 10.1134/S1063772909110092.

Lesovoi S., Kobets V. Correlation plots of the Siberian Radioheliograph. Solar-Terr. Phys. 2017, vol. 3, no. 1, pp. 19 25. DOI: $10.12737 / 23588$.

Lesovoi S.V., Altyntsev A.T., Kochanov A.A., Grechnev V.V., Gubin A.V., Zhdanov D.A., Ivanov E.F., Uralov A.M., Kashapova L.K., Kuznetsov A.A., Meshalkina N.S., Sych R.A. Siberian Radioheliograph: First Results. Solar-Terr. Phys. 2017, vol. 3, no. 1, pp. 3-18. DOI: 10.12737/article $58 / 96$ ec60/ec52.86165286.

Maksimov V.P., Nefedyev V.P. The observations of a "negative burst" with high spatial resolution. Solar Phys. 1991, vol. 136, no. 2, pp. 335-342. DOI: 10.1007/BF00146540.

Nakajima H., Nishio M., Enome S., Shibasaki K., Takano T., Hanaoka Y., Torii C., Sekiguchi H., Bushimata T., Kawashima S., Shinohara N., Irimajiri Y., Koshiishi H., Kosugi T., Shiomi Y., Sawa M., Kai K. The Nobeyama Radioheliograph // Proceedings of the IEEE. 1994, vol. 82, iss. 5, pp. 705-713. DOI: $10.1109 / 5.284737$.

Nakajima H.; Yokoyama T., A nonthermal collimated ejection observed with the Nobeyama Radioheliograph. Astrophys. J. Let. 2002, vol. 570, iss. 1, pp. L41-L45. DOI: $10.1086 / 340832$.

Pontieu B.De., Title A.M., Lemen J.R., Kushner G.D., Akin D.J., Allard B., Berger T., Boerner P., Cheung M., Chou C., Drake J.F., Duncan D.W., Freeland S., Heyman G.F., Hoffman C. The Interface Region Imaging Spectrograph (IRIS). Solar Phys. 2014, vol. 289, iss. 7, pp. 2733-2779. DOI: 10.1007/s11207-014-0485-y.

Raouafi N. E., Patsourakos S., Pariat E., Young P.R., Sterling A.C., Savcheva A., Shimojo M., Moreno-Insertis F., De-
Vore C.R., Archontis V., Török T., Mason H., Curdt W., Meyer K., Dalmasse K., Matsui Y. Solar coronal jets: Observations, theory, and modeling. Space Sci. Rev. 2016, vol. 201, iss. 1-4, pp. 1-53. DOI: 10.1007/s11214-016-0260-5.

Shimojo M., Hashimoto S., Shibata K., Hirayama T., Harvey K.L. Statistical study of solar X-ray jets observed with the YOHKOH soft X-Ray telescope. Astron. Soc. of Japan. 1996, vol. 48, pp. 123-136. DOI: 10.1093/pasj/48.1.123.

Uralov A. M., Lesovoi S. V., Zandanov V. G., Grechnev V.V. Dual-filament initiation of a coronal mass ejection: observations, model. Solar Phys. 2002, vol. 208, no. 1, pp. 69-90. DOI: 10.1023/A:1019610614255.

Uralov A.M., Grechnev V.V., Rudenko G.V., Myshyakov I.I., Chertok I.M., Filippov B.P., Slemzin V.A. A challenging solar eruptive event of 18 November 2003 and the causes of the 20 November geomagnetic superstorm. III. Catastrophe of the eruptive filament at a magnetic null point and formation of an opposite-handedness CME. Solar and Stellar Astrophys. 2014, vol. 289, iss. 10, pp. 3747-3772. DOI: 10.1007/s11207-014-0536-4.

Zirin N. The microwave brightness temperature spectrum of the quiet Sun. Astrophys. J. 1991, vol. 370, pp. 779-783.

URL: https://sohowww.nascom.nasa.gov/ (accessed November 23, 2017)

URL: https://stereo.gsfc.nasa.gov/ (accessed November 23, 2017).

URL: badary.iszf.irk.ru (accessed November 23, 2017).

URL: http://solar.nro.nao.ac.jp/ (accessed December 13, 2017).

URL: http://www.lmsal.com/solarsoft/irisa/ (accessed November 23, 2017 г.).

URL: https://kauai.ccmc.gsfc.nasa.gov/DONKI/search/ (accessed February 15, 2018).

URL: http://solar.nro.nao.ac.jp/norh/html/prominence (accessed March 5, 2018)

URL: https://hinode.isee.nagoya-u.ac.jp/ICCON/ (accessed November 23, 2017). 2017).

URL: http://ckp-rf.ru/usu/73606 (accessed November 23,

How to cite this article

Fedotova A.Yu., Altyntsev A.T., Kochanov A.A., Lesovoi S.V., Meshalkina N.S. Observation of eruptive events with the Siberian Radioheliograph. Solar-Terrestrial Physics. 2018. vol. 4, iss. 3, pp. 13-19. DOI: $10.12737 /$ stp-43201802. 